\title{
NIGEL HUNT: 'I WANTED TO BE AN ORTHODONTIST BEFORE I WANTED TO BE A DENTIST'
}

Professor Nigel Hunt has been Professor and Head of the Department of Orthodontics at the UCL Eastman Dental Institute since 1998. His clinical interest is the management of craniofacial deformity, and he leads a research team in the field of craniofacial reconstruction and tissue engineering. Nigel was elected Dean of the Faculty of Dental Surgery at the Royal College of Surgeons of England in June 2014. He is also the immediate past-Chairman of the British Orthodontic Society and its President for 2015.

\section{Why did you choose to} specialise in orthodontics?

I actually wanted to be an orthodontist before I wanted to be a dentist. Slightly unusual. At school I loved mechanical engineering. In fact I considered becoming an engineer but I also wanted to do something with health. Having then had orthodontic treatment myself I realised that orthodontics is mechanical engineering in many ways, whether you are moving teeth or reconstructing faces. So I was sold right at the beginning... even before I went to university I wanted to be an orthodontist.

\section{What are the challenges for specialist} dentistry in the future? We should really concentrate on the interaction between primary care and secondary or specialist care (whether on the high street or in hospital settings). We know that the Government, certainly NHS England, are keen to make sure that a number of the services which are currently provided in hospital care are moved into primary care. We must make sure that if this happens it is via appropriate care pathways with appropriate contracting arrangements. We need to make sure that people are properly trained and operating within their capabilities. This is certainly a challenge and in itself raises a whole range of issues, including: are there enough specialists working in primary care? Are there enough specialists full stop?
'We should really concentrate on the interaction between primary and secondary care...'

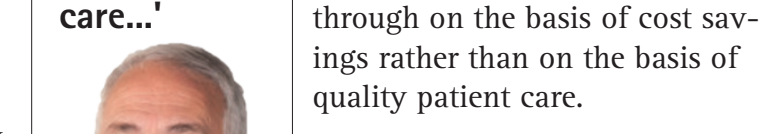

(क) systems to specialist care are working effectively in dentistry?

Looking at orthodontics as an example, far too many patients who are unsuitable for treatment are being referred currently. Either their oral hygiene is inappropriate or the practitioner hasn't spent enough time ensuring that the patient actually wants the treatment and is prepared to wear appliances. I will probably get shot down in flames for saying this but the system of remuneration at the moment means that some practitioners cherry pick. I'm emphasising 'some' here because there are an awful
lot of very good and fastidious specialist practitioners who will cover all sorts of complicated treatments quite rightly, but there are still some who will pick and choose. They are more inclined to refer those treatments which might take a longer period of time, such as treating impacted canines that need to be brought down into the mouth, into a secondary care setting when in fact they are more than capable and trained to do this themselves.

You are currently the 24th Dean of the Faculty of Dental Surgery at the Royal College of Surgeons - what are your main goals while in this position?

First of all, I don't think that we offer our members enough at the present time. We are too Londoncentric so one of my goals is to get more regional activity in terms of educational facilities and opportunities; in the UK but also internationally. We are in the process of trying to develop engagement with our members overseas and to get a better understanding of their educational needs.

On the policy side of things we need to focus particularly on engaging with government. We must emphasise that we are the voice of specialist dentistry so when it comes to making and designing policies we are right up there at the forefront, making constructive suggestions and being proactive rather than reactive.

\section{Do you feel dentistry and} medicine should be more closely integrated?

I do and I think they are coming together more now because of the recognition of the fact that oral health and general health are so inter-related. Personally, I would support the 'polyclinic' idea put forward by Lord Darzi. I think there is a space for medical as well as dental 
colleagues to work in the same environment. For example, you could have a diabetic clinic where within one building patients could see the specialists looking after their diabetes and vascular health, an eye specialist looking to see if they have any ocular problems and there could also be a periodontologist because of the relationship between periodontal disease and diabetes.

From the point of view of prevention, there is also a possibility of greater interaction because if you can get oral health sorted at an early age there is good evidence to show that it will be maintained for the rest of your life. There are other conditions too such as early detection of cancer where dental practitioners are ideally situated to examine patients' mouths and identify oral carcinoma, which if there is more integration between medicine and maxillofacial surgery can lead to a more effective management of patients.

\section{Is there a postcode lottery when it comes to the provision of dental services in the UK?}

This is definitely happening at the moment, particularly in multidisciplinary care situations, and it has got to be sorted out. I think part of the reason for this is since the abolition of PCTs we haven't really sorted out the new commissioning arrangements yet. An example of this is the provision of reconstruction of patients who have had oral cancer; for example, the availability of implants in post cancer reconstruction is so variable. We hear reports of patients attending one hospital and being turned down for a request for implants and then they are immediately accepted by another hospital just down the road because their terms of engagement are totally different. We see this not just locally but nationally. As practitioners, we are the ones who get the complaints from patients they don't complain to the commissioners directly. Dealing with these sorts of problems of patients being pushed from pillar to post is causing patient distress as well as taking up a lot of clinical time.

There are also examples where turning down treatments can cost more in the long term. For example, looking at orthognathic surgery, there are about roughly 3,000 cases undertaken in England and Wales per year but the cost is relatively minor. Several of these treatments can be done on almost an outpatient basis nowadays. If you turn these cases down, there is a lot of evidence to show that if patients have a poor quality of life they don't look after their teeth and that then means they are a burden on NHS dentistry for the rest of their lives. So, in terms of the overall cost, a relatively small amount now can have major lifelong benefits. This is really something that we need to get to grips with through discussion with NHS England and the government.

\section{You were highly involved in the design of the new Index of Orthognathic Functional Treatment Need (IOFTN). Why was this necessary?}

As I mentioned already there is a problem with commissioning and in my experience there is absolutely no point in complaining about a system which is not working unless you can come up with some positive suggestions to help. This was also appreciated by the British Orthodontic Society which led to development of the IOFTN.

The index of orthodontic treatment need (IOTN) is already applied nationally in both primary and secondary care environments and has been adopted by the commissioners to ensure that the funding for orthodontic treatment goes to the most needy patients. In a similar way, to try and help the situation with regards to orthognathic work, we developed the IOFTN to aid commissioners and referring practitioners by drawing up a scale of the various functional conditions for which patients may request orthognathic treatment, from very great need down to minimal and no need. It can be applied in the same way as the IOTN.

However, it is important to emphasise that the IOFTN only considers oral function and is only part of the overall assessment of the need of the patient. It should be used hand in hand with the psychological assessment as well. One of the problems with managing patients with facial deformity is the psychosocial problems which they experience. I've had 16-year-olds who have had major attempts at suicide because of teasing about their facial appearance. Their selfesteem and quality of life is rock bottom. However, quality of life is one aspect of healthcare that commissioners vary in their considered importance. Some have actively dismissed it as being unimportant in terms of healthcare. Yet the WHO definition of health includes social and psychological wellbeing. We can't dismiss it.

The next step with the IOFTN is to make sure that it is disseminated widely to both the profession and to the commissioners to make sure that: a) they are aware of it, and b) they can apply it.

What is your view on short-term orthodontics? I have no objection whatsoever to short-term orthodontics provided it's done by the right people for the

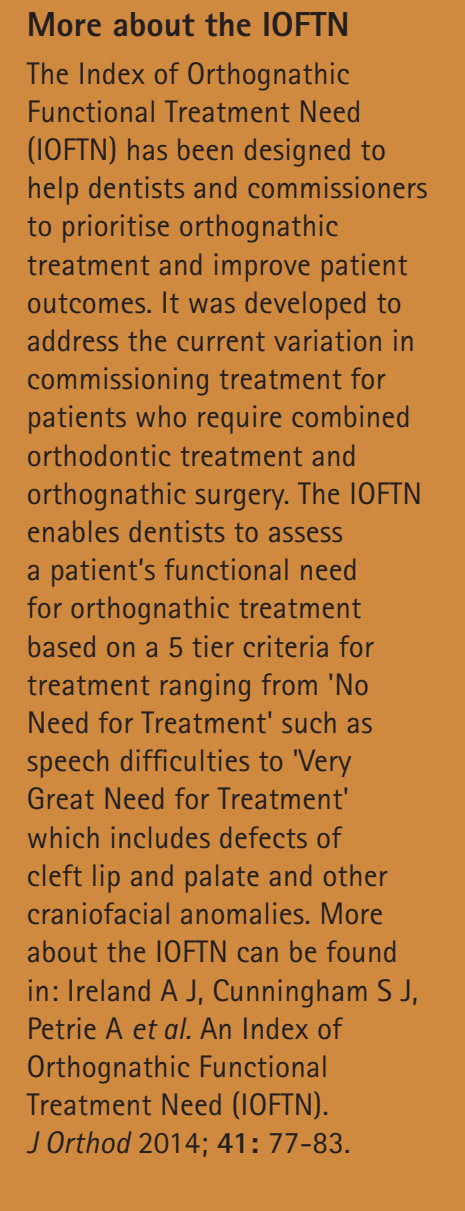

right reasons. Most importantly the patients must understand their options and I think that's where it has really come into disrepute. There are, of course, some extremely good general practitioners who are well trained and very sensible in terms of when they apply this treatment. They are doing their patients great service because they are avoiding what would otherwise be a monumental destruction of sound teeth, in oder to improve patients' appearance through crowns, veneers and so on. So from that point of view I have no qualms about it.

The single most important factor is that the patient must be given a full diagnosis of the whole problem. The range of options, together with the associated risks and benefits of each form of treatment, must be provided, including 'no treatment' because that is always a possibility. I think there have been instances where patients have been almost talked into treatment which is not necessary. It is also important that the clinicians who are providing the treatment are 
able to recognise when things are going wrong. I think one of the biggest reasons there has been an enormous rise in cases going to the indemnity societies is because of the fact that treatment hasn't worked out as expected and the person providing the treatment doesn't have the competence or the training to recognise it's going wrong and to know how to correct the problem.

There is also a big question about who should provide the training. I was recently involved in a working group with COPDEND for establishing a quality framework for CPD. This includes a checklist for what participants need to look for in terms of deciding whether a CPD course is a suitable training programme to undertake. I would be concerned that a trade company providing a two-hour training in a practice over lunch for the whole dental team is not really going to give you the comprehensive training required to embark on providing some of these treatments. I would question whether that is feasible by anybody - no matter how good a teacher or student they might be.

\section{What must we do to improve the general oral health of the population in the UK?}

Recognising the inter-relationship between oral health and general health is so important, as I've already mentioned. We have to get in young because if we have good oral health in children then that will manifest itself throughout life. There are still inequalities in terms of access eg the predominant reason why children are admitted to hospital in England is for extractions. If we can get that resolved it will ultimately reflect on general health in later life.

We need to focus on patient education and it's not just about encouraging people to go to the dentist; although as a Faculty, we'd like to see NICE guidelines implemented in terms of recommending one visit a year rather than every two years, as at present. We'd like to see better education for parents and carers, and also in schools. A lot of schools are offering sugar in various forms, either in lunch breaks as part of school meals or as treats or rewards for performance. Clearly, this is encouraging snacking throughout the day. As a Faculty, we are also lobbying for more local authorities to introduce water fluoridation as a means of reducing inequalities in children's oral health.

\section{What has been the proudest moment in your own career?}

Obviously the proudest moment was being elected as Dean of the Faculty here at the Royal College. Particularly, as coming from the Eastman, I am following in the footsteps of the Faculty's first Dean, Sir Robert Bradlaw.

On a personal level, I'm proud of the many students who have passed through the Eastman's orthodontic training programme. At this point in my career I can look back with pride that some students whose goal was to train as specialist orthodontists have now gone on to become senior academic clinicians. When they started they weren't entirely sure of their future but because of the way the programme is structured (it's a requirement to do a research masters degree) some individuals were stimulated to recognise that they wanted to follow an academic career. Seeing them now progress to the point where, for example, one is now a recognised professor in her own right at the Eastman is quite a proud moment. For example, Sue Cunningham has built up her own research portfolio where she is now recognised as being one of the world's leaders in the psychosocial aspects of malocclusion and facial deformity. To see that progression from a young non-specialist through to being at the pinnacle of her own career is fantastic.
What would you advise those considering specialising in orthodontics?

Do it! It is a very rewarding aspect of dentistry. Each patient is different. I find people fascinating. We've all got individual stories and the great thing about orthodontics is that you are largely treating people who want to be there. Also because treatment takes a relatively long period of time you do really get to know patients very well indeed. You almost get into their lives. Seeing the change that orthodontics can make to patients in terms of their confidence and self-esteem is so dramatic - you just can't quantify that. It was really a foregone conclusion that I wanted to do it.

\section{Three desert island books?}

- One that I would certainly take is a book by John Major. Not anything to do with politics at all but I'm an avid cricket supporter. In fact I'm a qualified cricket coach. He wrote a book called More than a game about the early beginnings of cricket and it just reminds me so much about the happy times I've either been at Lords as an MCC member or just coaching and playing cricket myself. I read it over and over again.

- Anything by Jeffrey Archer - complete escapism which allows me to just switch off and to read for the sake of reading.

- A History of English-speaking peoples by Winston Churchill. I'm cheating with this one because it's in four volumes. 